\title{
Safety Practices in the Lebanese Construction Market: Contractors' Perspective
}

\author{
R. Awwad ${ }^{\mathrm{a}}$, M. Jabbour ${ }^{\mathrm{b}}$ and O. El Souki ${ }^{\mathrm{c}}$ \\ ${ }^{a}$ Civil Engineering Department, Lebanese American University, Lebanon \\ ${ }^{b}$ Civil Engineering Department, Lebanese American University, Lebanon \\ ${ }^{c}$ Civil Engineering Department, Lebanese American University, Lebanon \\ E-mail: rita.awwad@lau.edu.lb, melanie.jabbour@lau.edu,omar.elsouki@lau.edu
}

\begin{abstract}
The construction industry in the world is considered to be one of the most dangerous industries, claiming each year the lives of many workers. In particular, the boom in the construction industry in the Middle East over the past decades was accompanied with significant increase in death tolls and injuries among labor forces. Lebanon is a small developing country in the region who survived a harsh civil war that ended in 1990, and since then, its economy has become more dependent on the construction industry. The country has been undergoing a reconstruction and rehabilitation phase that led to a prosperous growth in construction, however, not accompanied by any improvement of the construction laws to include safety regulations and standards. The purpose of this research is to assess the existence and extent of application of safety practices by Lebanese contractors, management commitment to safety, implementation challenges and opportunities for enhancing safe practices. Based on the aforementioned, this study highlights the benefits and deficiencies of the current safety practice in the Lebanese construction market and concludes by providing recommendations to positively influence industry professionals and foster their commitment to safety. The aim of this paper is achieved through conducting one to one interviews with Lebanese contractors. Findings showed a lack of awareness among small and medium size contractors about the necessity of developing and applying safety training programs on the jobsite. However, large contractors showed a much stronger commitment to safety through planned safety management programs and regular inspections but within a limited budget.
\end{abstract}

\section{Keywords}

Construction management, injuries, hazards, construction safety, construction worker, safety and health management systems, Lebanese construction market.

\section{Introduction}

The construction industry has been the leading cause of the most dangerous injuries among all other industries [1]. According to the U.S. Bureau of Labor Statistics in the U.S. department of Labor, the construction industry resulted in the most fatal injuries in 2012 [2]. The nature of work occurring on construction sites is hazardous since it involves the use of heavy equipment, erection of temporary structures extending to considerable heights, the use of unhealthy materials, as well as having to work sometimes in extreme natural conditions for lengthy time periods. Construction injuries not only have direct costs of treatment and compensation but they also have several indirect impacts on the workers such as low morale and loss of productivity. This has direct impacts on the project progress including completion delays, resource replacement costs, and higher insurance premiums. In order to remedy for this situation, safety measures and programs have been introduced and have even become required by law in some countries. In fact, a cost-benefit analysis of accident prevention conducted by Ikpe and et al. showed that the benefits of accident prevention outweigh the costs of accidents by a ratio of 3:1 [3].

Most companies in developed countries design their safety programs following OSHA standards however the main challenge remains to ensure that management and supervisory personnel are committed enough to enforce these guidelines and to provide adequate training to workers and educate them about the importance of safe working conditions [4]. It is 
important to realize that a safety program's success lies in the involvement of all concerned parties: the contractor, the owner, the designer, and the workers. Designers have a role in construction safety by including safety considerations in design details and specifications, ensuring contractor's submittals provide for worker's safety, and inspecting construction sites for safety [5]. Owners, as well, are involved in securing the safest working conditions through the selection of safe contractors, including clauses related to safety in contracts, participating in safety management and governing project characteristics [6, 7]. And, contractors constitute a key element in providing a safe jobsite through implementing safe construction techniques, providing personal protective equipment, training workers and supervisors on site, and improving their safety practices through injury record keeping and learning from previous experiences.

This paper presents an assessment of the existence and implementation of safety practices in the construction industry in Lebanon through interviews conducted with several contractors practicing in the market today. This survey sheds the light on awareness of Lebanese contractors about the importance of safety on site, the common types of injuries and provided insurances, the adopted safety standards if any, the role of managers and employees in monitoring safety practice, and concludes by providing recommendations to promote a safety culture within the construction industry. This research is the preliminary phase of a wider scale study that is being conducted by the authors to survey all involved market constituents including designers, owners, developers, insurance providers, and concerned public authorities in order to form a clear idea about the main challenges to safety implementations. This study will be eventually used to develop a simple and cost-effective construction safety program tailored to the Lebanese market risks and conditions which can positively influence industry professionals and convince them of the necessity of a safe construction site.

\section{The primitive safety culture in Lebanon and the surrounding region}

The term "Safety culture" was first discussed by the International Atomic Energy Agency as being one of the causes of the Chernobyl accident in 1986 [8]. Safety culture was then defined in various ways, however all definitions focus on the individuals' attitudes towards the safety system [9]. Construction safety culture is the combination of the beliefs, norms, attitudes and work practices aimed at reducing the vulnerability of workers and the public to accidents in the construction industry
[10]. Commitment to safety, awareness about its importance, and enforcement of corresponding legislative measures varies significantly among different construction industries. In the United States, the Occupational Safety and Health Administration (OSHA) sets and enforces construction work standards to ensure safety of the workplace and to protect health of the workers. The agency with the help of the United States Environmental Protection Agency (EPA) monitors the compliance with OSHA regulations through regular inspections of construction sites and punishment of violations. As for the European Union member states, the European Union law enacted construction safety principles in a main framework in 1989 (Directive 89/391/EEC) to encourage both public and private sectors to improve occupational health and safety and enforce a dual responsibility on the employer to provide safe working conditions and on the worker to adhere to safety instructions and report potential hazards [11].

However, this is not the case in the Middle East region in general and Lebanon in specific where the construction industry is booming and providing lot of employment opportunities to labor force without any considerable attention to the safety of the site nor to the health of workers. In the UAE, for instance, the federal law applicable to all seven emirates addresses health and safety in general but does not include any specific legislation dedicated to construction safety. A recent study by the UAE University found that two-third of hospitalized injuries occurring on building sites resulted from lack of or inappropriate personal protective equipment [12]. Similarly, the construction industry in Kuwait was characterized by lack of safety standards and weak implementation of labor safety rights through examining data for accidents on construction sites for the last ten years. Although Chapter XV of the Kuwaiti private sector labor law gives public officials the right to inspect and enforce the law, most contractors agreed that such inspections never took place [13]. Another study conducted about the Egyptian construction market showed that workers on site do not undergo any training programs or safety meetings. It further showed that most companies do not include safety in their planned budgets while very few others do but only within the range of 0.5 to $1 \%$ of the annual work volume [14].

Lebanon is a developing country that is still recovering from a 15-year civil war that ended in 1990 and after which the construction industry started booming exponentially. However, occupational health and safety was never given importance in that dynamic and hazardous field, and monitoring systems and relative legislation were never established by the government to control the inherent risks of that 
profession. The Lebanese workers' compensation law allows an injured worker on site full medical care, $75 \%$ of his daily salary since the time of injury and compensation in case of permanent disability or death. However, few construction companies purchase insurances tailored to protect workers and cover their injuries but rather the majority opts to provide an insurance policy for the whole worksite without naming individual workers or to pay from their accounts at the time of injury [15]. And here it is important to note that big portions of the labor force in the Lebanese construction sector are non-Lebanese who are hired on a daily basis which contributes to the negligence of employers in insuring them. From here stems the interest of this study in surveying current safety practices in the Lebanese construction industry through interviewing contractors to know more about their approach and commitment to an enhanced safety culture.

\section{Survey Scope and Findings}

The purpose of this paper is to assess the adoption of construction safety programs in the Lebanese construction industry. For this aim, a qualitative survey was conducted through interviews based on a questionnaire with fifteen construction companies. The interviewees occupied either the position of project manager or safety manager in their companies. All construction firms conduct work in Lebanon of which $33 \%$ have projects abroad as well. The fifteen interviewed companies have their headquarters based within the Lebanese territories. All firms are specialized in residential and commercial buildings with only three of them executing transportation projects as well.

The questionnaire was divided into nine parts which are the main safety areas identified by the Construction Industry Institute [16]: (1) Company Information: gathering background information about the company such as main scope of work, number of employees, whether or not it adopts a safety and health management system (SHMS), potential incentives to adopt a SHMS in case no one exists or information about SHMS if adopted; (2) Management Commitment: assessing if top management participates in safety operations; (3) Staffing for Safety: allocated human resources for construction safety purposes; (4) Safety Education: the existence or lack of training programs for workers; (5) Worker Involvement: participation of workers in enhancing safety regulations and training new workers; (6) Punishment/ Reward System: assessing if any rewarding system is in place to promote good safety practice and punish non-conforming behavior (7) Subcontract Management: Assessing whether safety performance is a criterion in the qualification and subsequent supervision of subcontractors. (8) Accident
Investigation: keeping accident records and investigation strategies (9) Drug and Alcohol Testing: determining whether workers are tested for alcohol or drug abuse.

\subsection{Safety and Health Management System (SHMS)}

As shown in Figure 1, 53\% of the interviewed contractors adopted a SHMS. All large companies and $40 \%$ of the medium sized companies developed a SHMS. As it was expected, none of the small contractors has developed a SHMS. This may be explained by the fact that small contractors spend on safety a higher proportion of their profits than medium and large size contractors do [3]. $85 \%$ of the companies with no SHMS have less than 2 projects per year while $62 \%$ of the companies with a SHMS have more than 5 projects yearly. This proves again the direct relationship between a company's annual turnover and adopting a safety and health management system.

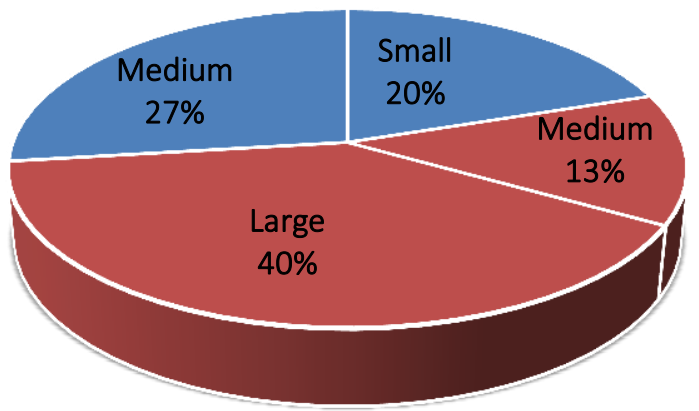

- Companies without SHMS

a Companies with SHMS

Figure 1. Distribution of companies by size and adoption of SHMS

By classifying the companies based on their number of service years, $54.5 \%$ of the companies with more than 10 years in the market have adopted a SHMS, as well as $50 \%$ of the companies who have now conducted construction business for 5-10 years. This shows that there exists no relationship between the age of the contracting company and its application of SHMS. It may be concluded that new companies are not shifting towards increasingly adopting SHMS despite the rise of awareness towards construction safety worldwide. One further observation is that all contractors working on international projects within the Middle East area have a SHMS, and only $30 \%$ of the companies solely working on local projects have a SHMS, all being large companies . 


\subsubsection{Companies without SHMS}

As reflected in Figure 2, 71\% of the companies with no SHMS claimed the reason to be that it is not required by law, $14.5 \%$ of them justified the absence of the SHMS through the high cost of safety implementation, and the remaining $14.5 \%$ answered that safety is a time consuming process. All respondents considered that the main incentive that would push them to develop and implement a safety program would be a strict legislative action by the government. All companies agreed that a citation in the range of $1,000,000$ L.L to $4,999,999$ L.L (\$667-\$3330) would lead them to adopt safe work practices as it would be more cost effective. It is worth noting that the government did release a decree in 2004 to deal with occupational safety, yet none of the interviewed contractors including the ones with SHMS had knowledge about.

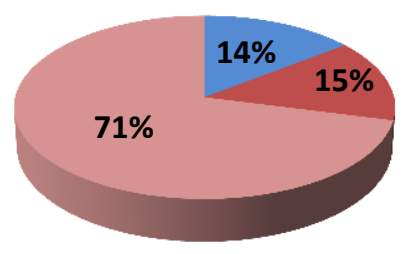

high cost of safety implementation

time consuming

not required by law

Figure 2. Reasons for lack of safety system adoption by contractors

\subsubsection{Companies with SHMS}

Companies based their SHMS either on Occupational Safety and Health Administration (OSHA) standards, requirements of the British Standards Institution (BSI), ISO 9001, on previous experience or on a combination of some or all. The tendency of construction companies to follow the OSHA standards $(50 \%)$ more than BSI standards $(25 \%)$ may be explained by the fact that American standards are viewed as less strict.

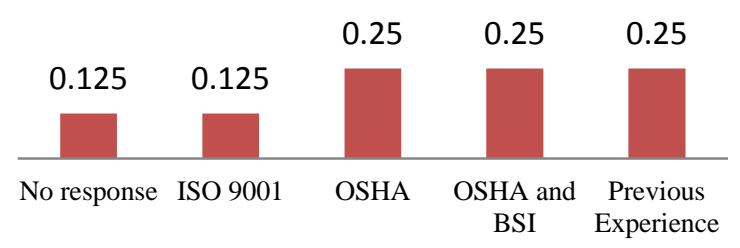

Figure 3. Safety standards adopted by Lebanese contractors with SHMS
Companies were asked about the main cause behind developing a SHMS. All respondents considered SHMS as an integral part of the company policy as opposed to being required by the owner or consultant. This implies that a company with SHMS will apply safety procedures even if not bound by contract.

The majority of companies gave priority to saving workers' lives $(63 \%)$ over saving the costs associated with a worker's injury or death, as shown in Figure 4. All these companies indicated that they continually update their safety and health management programs following changes in project phase, incident or accident on site, update in reference standards or new site requirements.

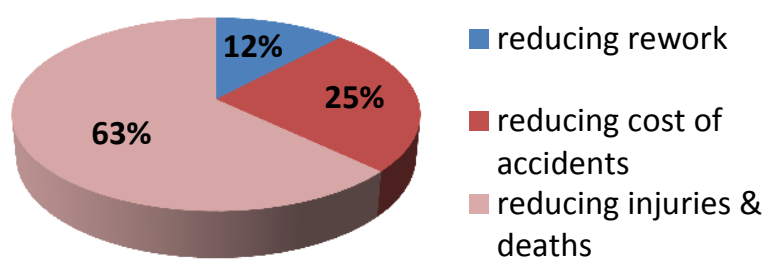

Figure 4. Reasons for adoption of safety standards by contractors

\subsection{Management Commitment}

Based on the responses of interviewees reflected in Figure 5, about half of them only spend more than $1 \%$ of their project budgeted cost on safety programs which may reflect a lack of full commitment and dedication to safe practice from the top management side. However, all interviewed companies having a SHMS organized regular site visits for top management and allocated corresponding resources such as meeting time, offices and tools for safety purposes. Even two among the firms who did not implement any SHMS had administrative personnel visibly participating through site visits. The administrative personnel's task was however limited to ensuring that no obvious unsafe behavior is present rather than checking the implementation of a fully developed safety system. Based on the aforementioned, it can be deduced that top management in general is willing to commit to safe work practices in terms of time and work, yet, they are not ready to allocate enough budget for this purpose.
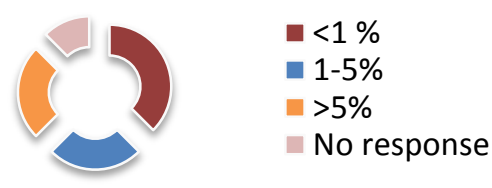

Figure 5. Percentage of project budget allocated to safety programs 


\subsection{Staffing for Safety}

This part of the survey deals with evaluating whether companies are allocating the required labor resources to properly implement safety procedures as part of the SHMS. It is obvious that companies with no safety and health management system reported having no Safety Officer (SO). As for the companies with a SHMS, seven out of eight had at least one full time SO. The company with no SO reported that the Project Manager (PM) is able to replace the SO. In order to confirm that the SHMS is properly implemented, site inspections are necessary. For instance, OSHA 1926 recommends regular inspections on Personal Protective Equipment (PPE), Personal Fall Arrest Systems (PFAS) and other protective equipment in addition to inspection on hazardous conditions similar to open excavations. Seven of the eight contractors with SHMS organized regular safety inspection and had allocated this task as part of the PM's job or staffed a safety inspector for this purpose. One company rented the services of a specialized inspection firm to ensure the complete implementation of its SHMS. The company with no safety inspector had a safety officer and considered that once the safety manuals are developed by the officer foreman, gangers, site engineers and PM will make sure of it being implemented without the need for inspections. Both companies who didn't staff a SO or a safety inspector had their SHMS based on personal experience and not on international standards. This may show the limitation of a safety program that is mainly based on experience from previous projects.

As part of accident management, contractors were asked whether they ensured the presence of a nurse or physician on site or at a nearby location. Obviously, none of the companies with no SHMS had a nurse or physician. Furthermore, two out of the eight contractors with SHMS staffed a nurse on site. One of the eight companies adopted a different approach by having an emergency team consisting of trained workers. The other five contractors didn't even have the knowledge nor considered the possibility of having a registered physician at a nearby location to promptly deal with accidents when they occur.

\subsection{Safety Education}

One of the most effective elements to reduce the risks of a certain activity is the safety and health training of all labor resources in a project [17]. Training falls within the safety education along with safety meetings and safety signs and regulations. The main aim of safety education is to make sure the workers are aware of the site hazards and to train them to effectively deal with those hazards. Seven of the eight companies with
SHMS provided training for their employees. The company which doesn't provide training for its employees had a SHMS based on its previous experience. This shows the necessity of having a national standard that companies should abide by rather than having a SHMS based on their own experience or on a collection of standards. Two of the companies with no SHMS did provide basic safety training for their workers. The training mainly covered either safe equipment operation or safe work procedures. Training was provided by foremen or site engineers based on their experience rather than safety manuals. As for companies with SHMS, the training covered a combination of hazards, work procedures, PPE, and safe handling of equipment.

Safety meetings are also an essential part of the SHMS. However, only seven of the eight companies organized safety meetings. Only five of the eight companies included workers in their safety meetings despite the fact that workers are an essential entity in safety meetings. All companies with SHMS posted safety regulations and signs in their job site to raise awareness towards hazards. None of the companies without SHMS had signs and regulations on site.

\subsection{Worker Involvement, Evaluation and Recognition, Drug and Alcohol Testing}

The questionnaire further assessed tradesmen involvement in the SHMS through participation in setting safety rules, accident investigation, and safety training. $75 \%$ of the companies with SHMS involved their workers in setting rules while only $50 \%$ involved them in accident investigation. In response to the inquiry about why workers are not involved in investigation, a contractor affirmed that workers lack objectivity as they may either fear top management or fully sympathize with affected worker. Three of the eight companies involved its workers in safety training of other employees; all three being large size companies. Moreover, two of the companies with SHMS recognized the safe performance of their employees through both monetary and verbal rewards; two other companies provided monetary reward while the remaining four didn't reward employees for applying safety rules. Even among the seven companies who lack a safety program, three of them did reward their employees for safe behavior. In case of safety related misconduct, all contractors with SHMS took measures up to termination of work. This shows a strict compliance to SHMS. Also, contractors without SHMS took measures against unsafe behavior as well; five of which reached job termination, one adopted decrease in monthly pay, and the last was limited to verbal warning. Although none of the 
interviewed companies tested its employees for drug or alcohol abuse neither upon employment nor during work, a written document banning drug and alcohol abuse existed in workers' contracts in $50 \%$ of the companies with SHMS. This shows the lack of awareness among Lebanese contractors about the importance of drug and alcohol testing for employees.

\subsection{Subcontract Management}

Hallowell and Gambatese affirm that subcontract management is among the most effective tiers of SHMS [18]. Only three companies with SHMS used safety performance as a prequalification criterion for the selection of subcontractors. However, all companies with SHMS bounded subcontractors by contract to follow safety procedures. This is not enough though to encourage subcontractors to be proactive in developing a safety culture and to be committed to safety training and education. As for companies with no SHMS, none of the companies considered safety neither in choosing subcontractors nor in contract with the latter.

\subsection{Accident/Incident Investigation}

As accidents and incidents indicate deficiencies in the SHMS, record keeping is essential to continually improve safety program and performance. All companies with SHMS kept record of accidents and incidents: six of which kept records for more than five years beyond the project completion date while the remaining two kept them for the duration of the project only. As for the seven companies without SHMS, only two kept records for more than five years and one for the duration of the project. Besides, only one of all interviewed companies which had no SHMS did not conduct investigation for causes of accidents when they occurred. Mostly the parties responsible for investigation were mainly the insurance representative assisted by the PM (in companies with no SHMS) or by the PM and Safety Officer (in companies with SHMS). This shows that companies with SHMS are serious about gathering data for the continuous update of their safety systems.

In response to the question regarding the most frequent type of injuries, nails in the foot and cuts were reported six times; eye splinters and fall from elevation appeared four times and object falling on head appeared twice. Having the fall from elevation as one of the most frequent accidents reveals the necessity of immediate action to enhance safety in the Lebanese construction industry.
Regarding the insurances obtained by all interviewed companies, they varied between all-risk, worker's compensation and third party liability insurances. Almost all companies provided a combination of these except three of the companies with SHMS who purchased all three types simultaneously.

Among the eight companies with SHMS, seven reported conducting work site analysis and updating it in order to deal with job specific hazards. This shows that the majority of those companies is not following a predefined template but is being proactive in preventing accidents. As for contractors with no SHMS, only 57\% conducted work site analysis which shows that PMs are improvising in the situation where no safety program exists to limit on site hazards.

\section{Conclusion}

Based on the presented survey findings, it can be concluded that there is a promising effort on behalf of mostly large Lebanese contracting companies to address safety concerns in their organizational policies through following international safety standards such as OSHA and BSI with continuous follow-up and updates. They also reported training workers regularly, assigning safety officers and inspectors on site to make sure their safety programs are implemented correctly, posting safety regulations and signs in their jobsites, and keeping track of accidents and investigating their causes. However, and although these companies showed concern for construction injuries and commitment to safe practices, they had allocated a low budget for safety in their projects, missed some other safety measures such as rewarding workers for compliance with safety and running alcohol and drug abuse tests on workers. Besides, $25 \%$ of these companies based their safety programs on previous experience which calls for the importance of drafting and legislating national safety standards that can be tailored to the Lebanese construction hazards and that can be applied homogenously across all construction firms, which would facilitate the process of monitoring compliance with those standards and penalizing violations.

On the other hand, the majority of medium and small construction companies did not have any safety management systems implemented on their job sites, neither safety officers nor trainings or signs. These firms reduced the whole safety program to just mere visits by administrative personnel in order to check that no unsafe practices are occurring during the course of work. These firms, however, showed willingness to implement safety regulations if they were enforced by law. And this highlights the role of the public health sector and all other relevant public authorities in raising 
awareness about construction safety among all industry constituents through establishing formal monitoring bodies whose function is to ensure implementation of safety practices on construction sites, to regularly inspect workers and keep track of work-related injuries, and to devise a set of significant violation fines that would push contractors to adhere to the safety law.

Last but not least, coordination and commitment of all construction parties including owners, designers, contractors, subcontractors, managers, site engineers, and workers is crucial to the success of any safety program and necessary to face any safety implementation challenges in the construction industry.

\section{References}

[1] Rosenfeld, Y., Rozenfeld, O., Sacks, R. and Baum. Efficient and timely use of safety resources in construction. In Proceedings of the CIB W99 2006 International Conference on Global Unity for Safety and Health in Construction, pages 290-297, Beijing, China, 2006.

[2] U.S. Bureau of Labor Statistics, U.S. Department of Labor, 2013.

[3] Ikpe, E., Hammon F., and Oloke D. Cost-Benefit Analysis for Accident Prevention in Construction Projects. Journal of Construction Engineering and Management, 138(8): 991-998, 2012.

[4] Abduyyeh, O., Fredericks T., Butt S., and Shaar A. An investigation of management's commitment to construction safety. International Journal of Project Management, (24): 167-174, 2006.

[5] Toole, T. M. Increasing Engineers' Role in Construction Safety: Opportunities and Barriers. Journal of Professional Issues in Engineering Education, 131(3): 199-207, 2005.

[6] Samelson, N. and Levitt, R. Owner's Guidelines for Selecting Safe Contractors. Journal of the Construction Division, 108(4): 617-623, 1982.

[7] Huang, X. and Hinze, J. Owner's Role in Construction Safety. Journal of Construction Engineering and Management, 132(2): 164-173, 2006.

[8] International Atomic Energy Agency. Summary report on the post-accident review meeting on the Chernobyl accident. Safety Series 75-INSAG-1, International Safety Advisory Group, Vienna, Austria, 1986.
[9] Choudhry, R., Fang D., and Mohamed S. Developing a Model of Construction Safety Culture. Journal of Management in Engineering, 23(4): 207-212, 2007.

[10] Zou, P. Fostering a Strong Construction Safety Culture. Leadership and Management in Engineering (11):11-22, 2011.

[11] EuroStat. Health and safety at work statistics. Online:http://epp.eurostat.ec.europa.eu/statistics_explai ned/index.php/Health_and_safety_at_work_statistics. Accessed: 14/11/2013.

[12] Kenrick V. PPE and Construction Safety in the Middle East. EHS Today Magazine, February 2012.

[13] Al-Humaidi, H. and Tan F. Construction Safety in Kuwait. Journal of Performance of Constructed Facilities , 24(1): 70-77, 2010.

[14] El Safty A. and Malek M. Construction Safety and Occupational Health Education in Egypt, the EU, and US Firms. Open Journal of Civil Engineering (2): 174-182, 2012

[15] Fayad R., Nuwayhid I., Tamim H., Kassak K. and Khogali M. Cost of work-related injuries in insured workplaces in Lebanon, Bulletin of the World Health Organization , 81 (7) :509- 515, 2003.

[16] Construction Industry Institute. Safety plus: Making zero accidents a reality. CII Project Rep. 160, Austin, TX, 2002.

[17] Hallowell, M. and Gambatese J. Population and Initial Validation of a Formal Model for Construction Safety Risk Management. Journal of Construction Engineering and Management, 136 (9): 981-990, 2010.

[18] Hallowell, M. and Gambatese J. Construction Safety Risk Mitigation. Journal of Construction Engineering and Management, 135(12): 316-323, 2009. 